\section{Modelling and simulation tests of a quadrocopter flying robot}

\author{
Wojciech Ciesielka, Grzegorz Suchanek \\ AGH University of Science and Technology, Poland
}

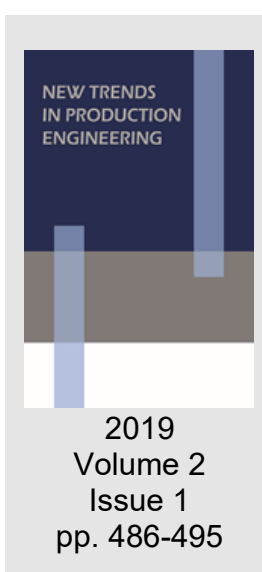

Date of submission to the Editor: 05/2019

Date of acceptance by the Editor: 08/2019

\title{
INTRODUCTION
}

Multirotor robots are used to take different measurements from the air because of their possibility of hovering anywhere. With the help of a thermal imaging camera, it is possible to search for vehicles in situations where visibility is limited by fog (Shamsi et al., 2018). Acoustic measurements (localization) are also possible (Wang and Cavalro, 2018).

An example of the use of multi-rotor robots for environmental measurement purposes is presented in the paper (Wivou et al., 2016). On the robot, an Arduino based measuring system was installed to measure the concentration of several example gases. As another example, work (Degner and Ewald, 2018) can be quoted. There, a mobile system for the detection of exhaust pollutants has been presented. The measurements are made using an spectroscopy based electrooptical system, utilizing LEDs and photodiodes. The system was installed on a commercially available DJI Phantom 4 robot.

However, in order to carry out reliable measurements, it is necessary to conduct advanced modelling and simulation tests of the flying platform. It also includes the analysis and determination of the appropriate location for the measurement system. Most of the current work on methods and techniques of taking measurements on the flying robots does not take into account this issues. Preliminary information on how to locate the measurement system is described in the paper (Bolla et al., 2018). In this work, this problem of the need to place the measurement system outside the turbulence zone was indicated. As the authors wrote, they also carried out some CFD simulations in the work and they found out that the measurement of particulate matter is possible only in the hovering state of the robot.

However, a common weakness can be indicated in the cited papers. It is related in the lack of the integration of the measuring system with the control system. This is due to various reasons: the use of commercial robots by the authors, or the inability to modify the flight controller software. The integration of these two, control and measurement subsystems, allows the robot to take active actions. 
For example, it is possible to search for a source of pollution, follow it or escape from the zone of over intensified pollution. The authors of this work designed, constructed and carried out verification tests of the original quadrocopter flying platform. The construction and field tests were presented in the papers (Suchanek and Ciesielka, 2018, Ciesielka and Suchanek, 2017).

The purpose of this work was to develop the mathematical models of a quadrocopter flying robot and also to determine the location of the inlet of a particulate measurement (PM) measurement system.

\section{THE QUADROCOPTER MATHEMATICAL MODEL}

As part of the first stage of the work, a mathematical model of the robot was developed. For this purpose, Euler-Lagrange equations were used. The coordinate system and the way of determining the order of the motors in this work were consistently assumed as in previous works (Suchanek and Ciesielka, 2018, Ciesielka and Suchanek, 2017). The schematic of the quadrocopter robot is presented on Figure 1.

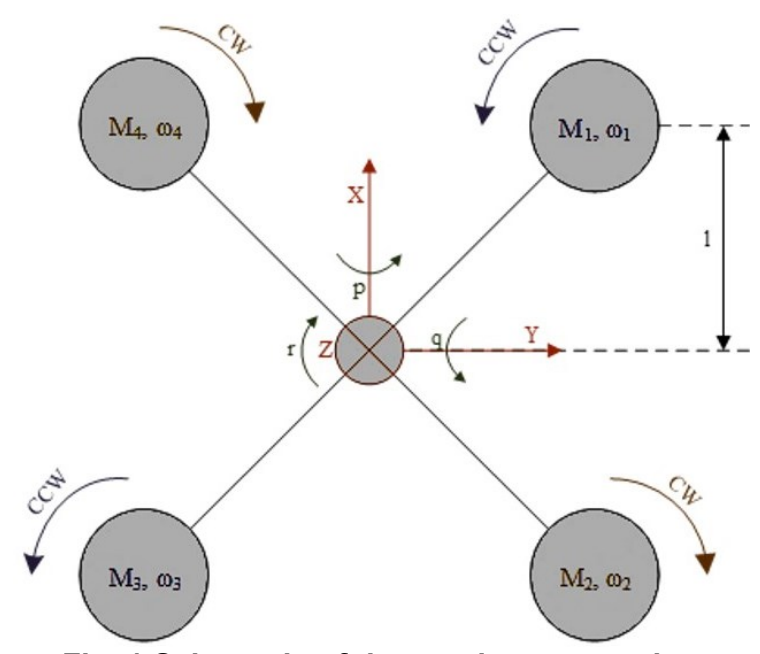

Fig. 1 Schematic of the quadrocopter robot

The robot is equipped with four rotors, marked $1 \div 4$, in clockwise sequence. The first motor is at right front ot the robot. The motors 1 and 3 turns to the left, and the motors 2 i 4 turns to the right. Each motor rotates at $\omega_{i}$ velocity, thus generate the $M_{i}$ torque and $F_{i}$ thrust force. The coordinate system is a standard NorthEast-Down system, where the $x$ axis is facing forward in the direction of flight. The quadrocopter rotates in its local system with the velocites $p, q$ i $r$ in the $x, y$ i $z$ axes respectively. In the global system, its translational movement position is represented by the $x, y$ i $z$ coordinates, and the angular position by a $\Theta$ (x axis), $\Phi$ (y axis), $\Psi$ (z axis).

The thrust force $F_{i}$ and torque $M_{i}$ generated by the each motor can be described as:

where:

$$
F_{i}=k \omega_{i}^{2}, M_{i}=b \omega_{i}^{2}+I_{M} \dot{\omega}_{i}
$$

$k, b$ - motor constants, $I_{M}$ - motor moment of inertia (negligible). 
On this basis forces $F_{\zeta}$ acting on the quadrocopter and torques $I_{\eta}$ coming from the motors (both depending of the rotational speed $\omega_{i}$ ) can be written as:

$$
\boldsymbol{F}_{\xi}=\left[\begin{array}{c}
F_{x} \\
F_{y} \\
F_{z}
\end{array}\right]=\left[\begin{array}{l}
u\left(-S_{\Phi}\right) \\
u\left(S_{\Theta} C_{\Phi}\right) \\
u\left(C_{\Theta} C_{\Phi}\right)
\end{array}\right]
$$

where:

$$
\begin{aligned}
& u=\sum_{i=1}^{4} F_{i} \\
& \boldsymbol{\tau}_{\eta}=\left[\begin{array}{c}
\tau_{\Theta} \\
\tau_{\Phi} \\
\tau_{\Psi}
\end{array}\right]=\left[\begin{array}{cccc}
-k l & -k l & k l & k l \\
k l & -k l & -k l & k l \\
b & -b & b & -b
\end{array}\right]\left[\begin{array}{c}
\omega_{1}^{2} \\
\omega_{2}^{2} \\
\omega_{3}^{2} \\
\omega_{4}^{2}
\end{array}\right]
\end{aligned}
$$

The symbol / indicates half of the distance between the motors axes (Fig. 1). Skipping the complex process of deriving individual motion equations, the final form of equations of the quadrocopter flying robot motion is presented below:

$$
\begin{gathered}
\ddot{x}=\frac{1}{m} F_{x} \\
\ddot{y}=\frac{1}{m} F_{y} \\
\ddot{z}=\frac{1}{m} F_{z}-g \\
\ddot{\Theta}=\frac{1}{I_{x x}}\left(\tau_{\Theta}-\frac{1}{2} \dot{\Phi}\left(I_{y y}-I_{z z}\right)\left(\dot{\Phi} S_{2 \Theta}+\dot{\Psi} C_{\Phi}-2 \dot{\Psi} C_{\Phi} C_{\Theta}{ }^{2}\right)+\frac{1}{2} \dot{\Psi}\left(I_{y y}-\right.\right. \\
\left.\left.I_{z z}\right) C_{\Phi}\left(2 \dot{\Phi} C_{\Theta}{ }^{2}-\dot{\Phi}+2 \dot{\Psi} C_{\Phi} C_{\Theta} S_{\Theta}\right)+I_{x x}\left(\ddot{\Psi} S_{\Phi}+\dot{\Phi} \dot{\Psi} C_{\Phi}\right)\right) \\
\ddot{\Phi}=\frac{1}{\left(I_{y y} C_{\Theta}{ }^{2}+I_{z z} S_{\Theta}{ }^{2}\right)}\left(\tau_{\Phi}+\frac{1}{2} I_{x x} \dot{\Psi}{ }^{2} S_{2 \Phi}-I_{x x} \dot{\Theta} \dot{\Psi} C_{\Phi}-I_{z z} \dot{\Psi}^{2} C_{\Phi} C_{\Theta}{ }^{2} S_{\Phi}-\right. \\
I_{y y} \dot{\Psi}^{2} C_{\Phi} S_{\Theta}{ }^{2} S_{\Phi}+\quad-\left(I_{z z}-I_{y y}\right)\left(\dot{\Theta} \dot{\Phi} S_{2 \Theta}+\dot{\Theta} \dot{\Psi} C_{\Phi} S_{\Theta}{ }^{2}+\dot{\Phi} \dot{\Psi} C_{\Theta} S_{\Theta} S_{\Phi}\right)- \\
\left.\left(I_{y y}-I_{z z}\right)\left(C_{\Theta} C_{\Phi} S_{\Theta} \ddot{\Psi}+\dot{\Theta} \dot{\Psi} C_{\Phi} C_{\Theta}{ }^{2}\right)\right) \\
\ddot{\Psi}=\frac{1}{\left(I_{x x} S_{\Phi}{ }^{2}+I_{y y} S_{\Theta}{ }^{2} C_{\Phi}{ }^{2}+I_{z z} C_{\Theta}{ }^{2} C_{\Phi}{ }^{2}\right)}\left(\tau_{\Psi}+I_{x x}\left(S_{\Phi} \ddot{\Theta}+\dot{\Theta} \dot{\Phi} C_{\Phi}-\dot{\Phi} \dot{\Psi} S_{2 \Phi}\right)-\right. \\
\quad\left(I_{y y}-I_{z z}\right)\left(C_{\Theta} C_{\Phi} S_{\Theta} \ddot{\Phi}+\right. \\
\left.I_{y y}\right)\left(\dot{\Phi}^{2} C_{\Theta} S_{\Phi} S_{\Theta}+\dot{\Theta} C_{\Phi} C_{\Theta}{ }^{2}+2 \dot{\Theta} C_{\Phi} S_{\Theta}{ }^{2}\right)+2 \dot{\Phi} \dot{\Psi}\left(I_{z z} C_{\Theta} C_{\Phi}{ }^{2} C_{\Phi}{ }^{2}\right)-\left(I_{\Phi}-\right.
\end{gathered}
$$

\section{THE DYNANOMETER MOBILE STATION}

In order to determine the necessary parameters of quadrocopter drive sets, a dynamometer mobile station was designed, constructed and programmed. The multicopter drive set is understood as a complete set consisting of: propeller, motor and electronic speed controller (ESC). The station allows to take measurements of thrust force, torque, rotational speed, current and supply voltage of the motor, temperature of its base and also vibrations (accelerations) in all three axes. The basis of the construction is a $0.3 \mathrm{~m} \times 0.3 \mathrm{~m}$ wooden board with a thickness of $18 \mathrm{~mm}$. The other elements are made of the aluminum. The height of the dynamometer is $0.342 \mathrm{~m}$.

The main element of the electronic part of the station is the NXP LPC1763 microcontroller, with the Cortex-M3 core, clocked at $100 \mathrm{MHz}$. Two HX711 load cell amplifiers from AVIA Semiconductor are used to amplify and convert to a 
digital form signal from the load cels. A bar with a permissible load of $5 \mathrm{~kg}$ was used to measure the thrust force, and a $1 \mathrm{~kg}$ bar to measure the torque. The motor current is measured using the ACS711 current to voltage transducer from Allegro Microsystems, enabling the measurement of current up to $25 \mathrm{~A}$. The measurement of the supply voltage, voltage from the current sensor and the LMT87 (accuracy $\pm 0.4^{\circ} \mathrm{C}$ ) temperature sensor was realized using the 12-bit ADC converter embedded in the microcontroller. The vibration measurement was carried out using a 16-bit 3-axis accelerometer embedded in the TDKInvensense MPU9250 sensor. The measurement of the rotational speed of the propeller is carried out using an optical barrier. Measurement data are collected in external 23LC1024 RAM memory with a capacity of $128 \mathrm{kB}$.

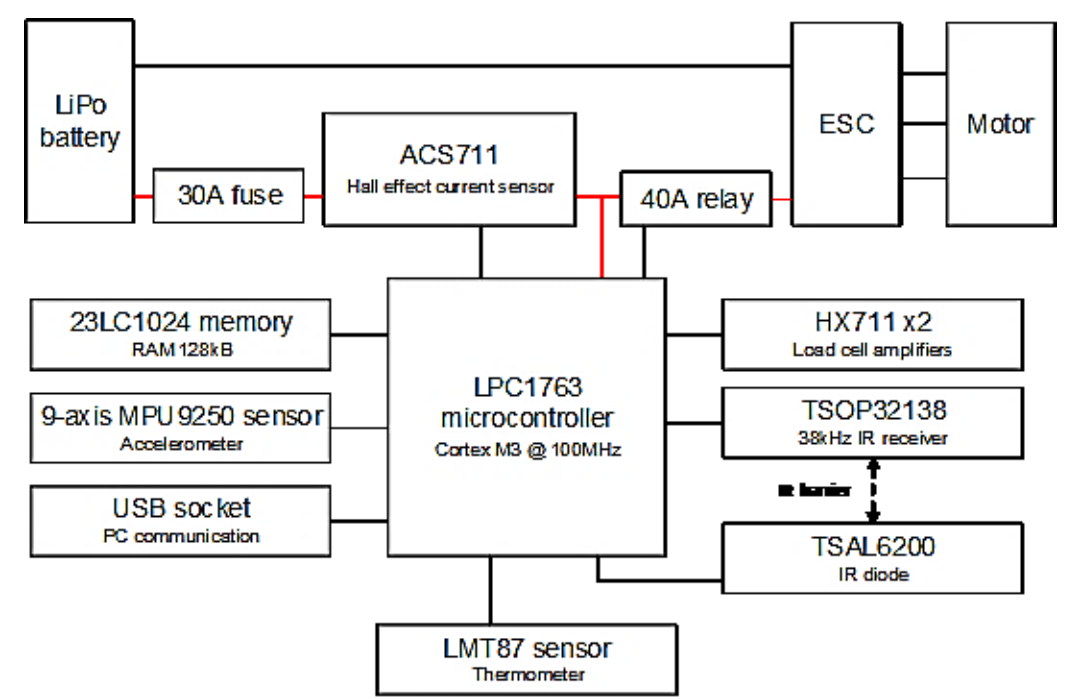

Fig. 2 The block diagram for the station

The possibility of communication of the station with a PC computer was ensured by using the FT232RL chip, an integrated USB-UART converter. To provide the necessary safety measures, the dynamometer was equipped with a standard car relay. The relay disconnects the motor supply in the event of the losing communication with the PC. The system also uses an additional safety switch to remotely disconnect the relay coil. The block diagram of the station is presented on Figure 2.

After performing the necessary calibrations, it was possible to measure, and then compare the engine performance for constant operating conditions with different propellers. The results of the research are presented on Figures 3 and 4.

On this basis, it was possible to choose a propellers with the best performance, which turned out to be a Gefman ABS/Carbon 1045 propellers. 


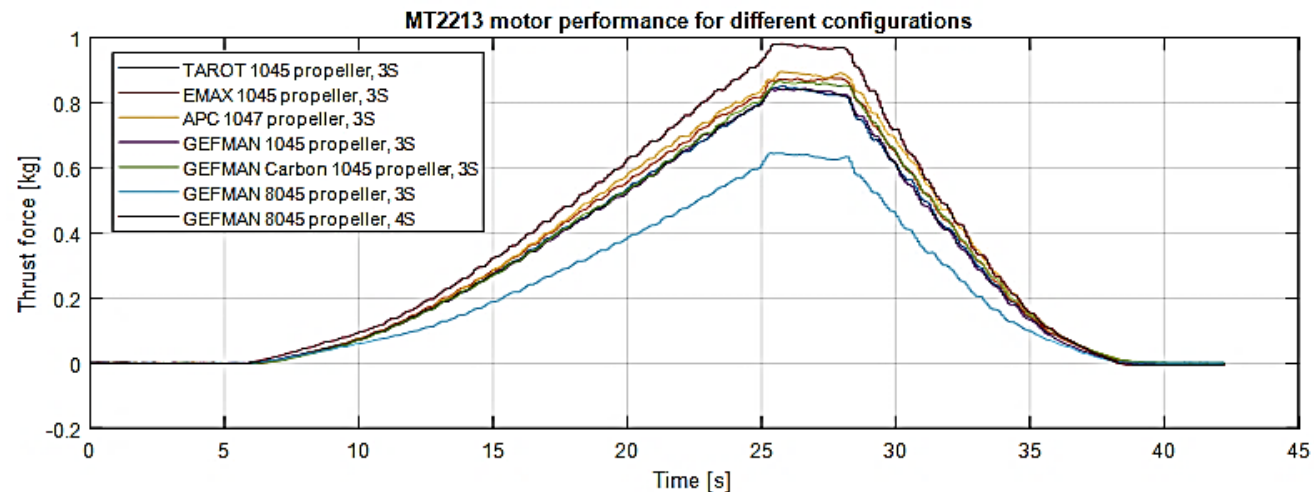

Fig. 3 MT2213 motor performance for different propellers (thrust force)

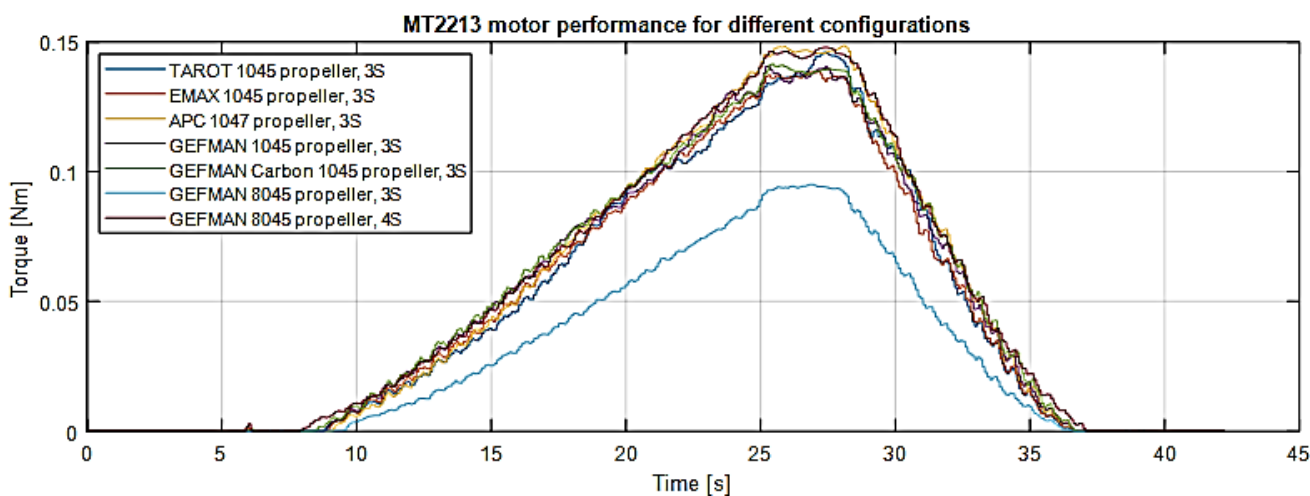

Fig. 4 MT2213 motor performance for different propellers (torque)

\section{THE COMPUTER MODELLING}

The developed mathematical model was implemented in the Matlab/Simulink environment. Due to its significant complication, the only the results of computer simulations will be presented.

The following parameters were used for the simulation: the total weight of the robot $m=1.3 \mathrm{~kg}$, gravitational acceleration $g=9.80665 \mathrm{~m}^{2} / \mathrm{s}$, the half of the distance between the motors axes (Figure 1) $I=0.16 \mathrm{~m}$. Based on the measurements carried out on the dynamometer station for the selected propellers the motor constants are: $k=1.79 \cdot 10^{-5} \mathrm{Ns}^{2}$ and $b=3.12 \cdot 10^{-7} \mathrm{Nms}^{2}$. The moments of inertia of the robot in relation to the center of gravity were determined analytically to: $I_{x x}=0.017 \mathrm{kgm}^{2}, I_{y y}=0.017 \mathrm{kgm}^{2}$ and $I_{z z}=0.015$ $\mathrm{kgm}^{2}$. On this basis, it was possible to determine the theoretical motors speed $n_{\text {hover }}$ in the robots hover condition, when gravity and total thrust force $u$ are balanced:

$$
n_{\text {hover }}=\sqrt{\frac{\frac{m g}{4}}{k}} * \frac{60}{2 \pi}=4029[\mathrm{rpm}]
$$

Based on the motors speed in the hover condition, the following curve, presented on Figure 5, was used in the simulation of the upward flight maneuver. This maneuver is obtained by changing the rotational speed of all engines simultaneously, in this case by a $60 \mathrm{rpm}$. 


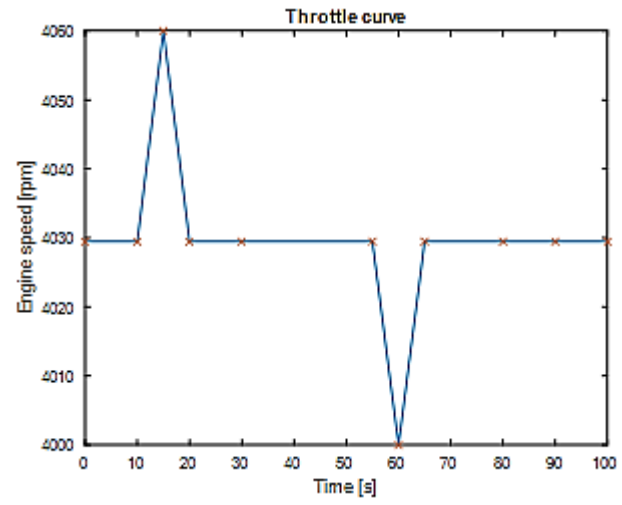

Fig. 5 The motors speed curve in the upward flight maneuver

Figure 6 presents the behavior of the robot in this maneuver. The robot rises upward in the $Z$ axis without changing its angular orientation.
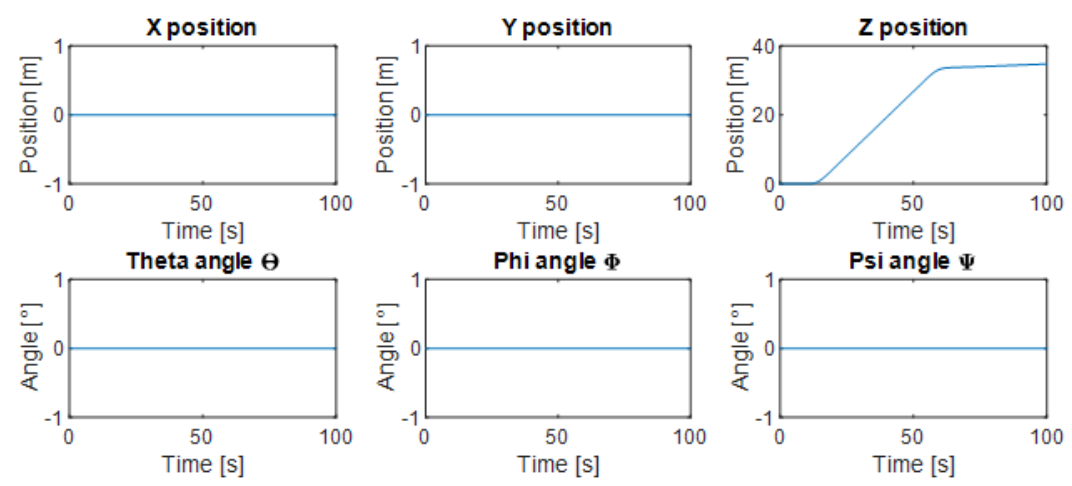

Fig. 6 The upward flight maneouver
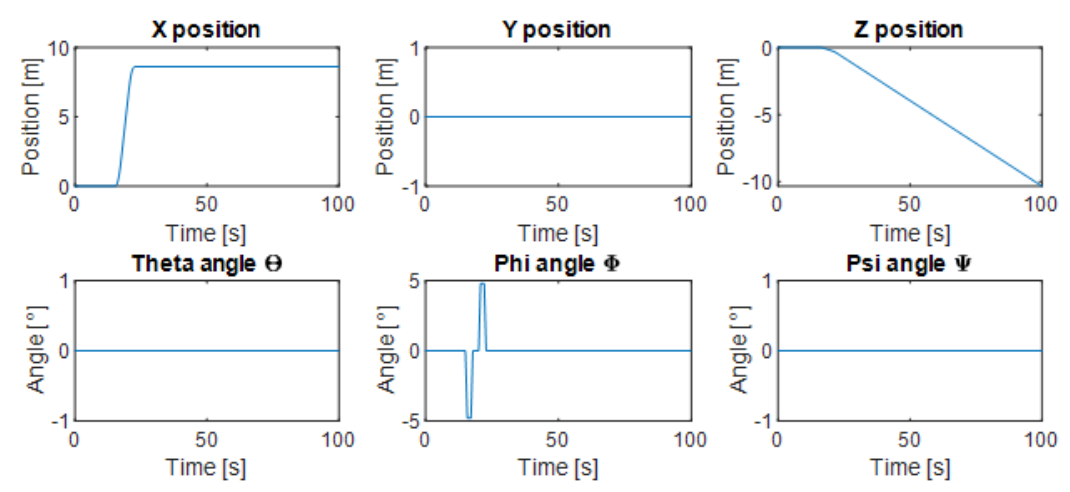

Fig. 7 The forward flight maneouver

The forward flight maneuver (Figure 7 ) is performed by increasing the rotational speed of the rear engines (2 and 3 ), and reducing the front engines ( 1 and 4 ) respectively. In this case, by a $30 \mathrm{rpm}$ relative to the hover condition. The robot rotates in the $Y$ axis, and thus at the same time moves in the $X$ axis. However because the robot is tilted, and the total thrust force $u$ are not acting strictly down, the robot also loses altitude.

\section{THE CFD MODEL FOR QUADROCOPTER}

After confirming the correct operation of the discrete model implemented in the Matlab/Simulink environment, the discrete-continuous model of the 
quadrocopter was developed. The work began with the 3D geometric model of the selected propeller. The 3D scanning process resulted in a polygon mesh (STL) file. The initial processing consisted in proper orienting of the solid in the coordinate system and simplification of the rough surface on the longitudinal sections. This part of the work was done in the SpaceClaim software included in the ANSYS package. Then, it was possible to further simplify the model body in the CATIA software.

The CFD model was built in the Workbench software of the ANSYS package. The prepared propeller was loaded into the geometry module, which was then placed in a cylinder with a diameter of $0.3 \mathrm{~m}$ and a height of $0.1 \mathrm{~m}$. This cylinder is a rotating domain. Then, three additional rotating domains with propellers were created using the translation and mirror tools into the desired configuration (alternating left and right propellers). The four rotating domains was then enclosed in a static zone, in the shape of a rectangular cuboid measuring 0.75 $\mathrm{m} \times 0.75 \mathrm{~m} \times 0.20 \mathrm{~m}$. The final step was to subtract the volume of all cylinders from the static zone, and then each of the propeller bodies from the corresponding rotational domain, using the boolean substract function.

In the next step, a non-structural mesh was prepared in the Mesh module. For this purpose, it was necessary to estimate the height of the first boundary layer. To determine this parameter, the following assumptions were made:

- Propeller rotational velocity $N=4029 \mathrm{rpm}$,

- Propeller diameter $d=10$ inches $=0.254 \mathrm{~m}$,

- Air density $\rho=1.25 \mathrm{~kg} / \mathrm{m}^{3}$,

- Air viscocity $\mu=1.78 \cdot 10^{-5} \mathrm{~m}^{2} / \mathrm{s}$,

- Desired $y+$ value $=1$.

Based on analytical calculations, the height of the first boundary layer $D S$ was estimated to $0.051 \mathrm{~mm}$. However, based on further experiments, it was necessary to decrease the $D S$ parameter to $10^{-5} \mathrm{~m}$ (that is $0.01 \mathrm{~mm}$ ) to obtain the $y$ + parameter at 1.26. The total of 5 boundary layers were assumed for each rotor, and the inflation rate was set to 1.2. In addition, it has been defined so that the size of the element in the rotating domain does not exceed $4 \mathrm{~mm}$, and in the static zone $30 \mathrm{~mm}$. The total number all elements in a prepared mesh is 6574140.

The kw-SST turbulence model was used for calculations. After completing 328 steps of simulation for the steady state, the results converged at a $10^{-3}$ accuracy. As part of this stage of the work, the resultant thrust forces and torques for each propeller were determined. For each of them, a thrust force of $2.78 \mathrm{~N}$ was obtained, which sums to a total of $11.08 \mathrm{~N}$ for four propellers. The total thrust force in $\mathrm{kg}$ for a simulated model in the hover condition is $1.13 \mathrm{~kg}$. This result is less by $13 \%$ than the robot weight of $1.3 \mathrm{~kg}$. A similar difference occurs for the resultant torques, where the $0.048 \mathrm{Nm}$ was obtained. In this case, the moments for the left propellers (turning $\mathrm{CCW}$ ) are negative and for the right propellers are positive. 
The most important part of this CFD research was the determination of velocity and pressure fields distribution in static and dynamic domains.

Figure 8 shows the air velocity magnitude on the suction and discharge sides. Figure 9 presents the dynamic pressure on the suction and discharge sides. Figure 10 shows air velocity magnitude and dynamic pressure on the side surfaces.

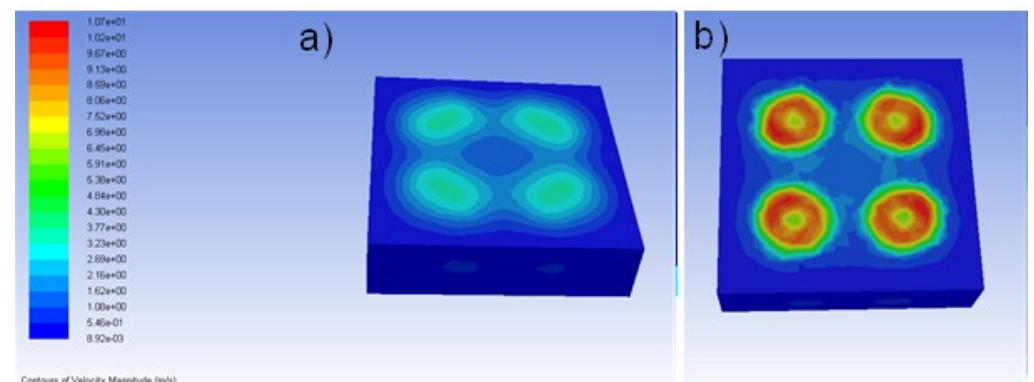

Fig. 8 The air velocity magnitude: a) suction side, b) discharge side

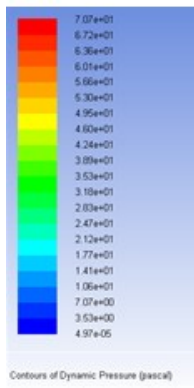

a)

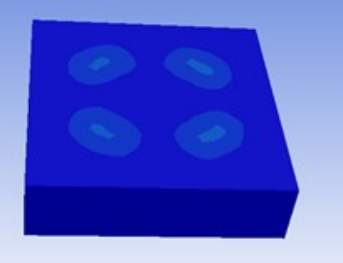

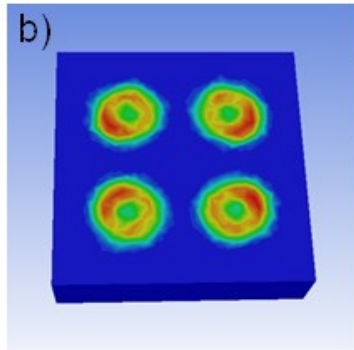

b)

Fig. 9 The dynamic pressure: a) suction side, b) discharge side
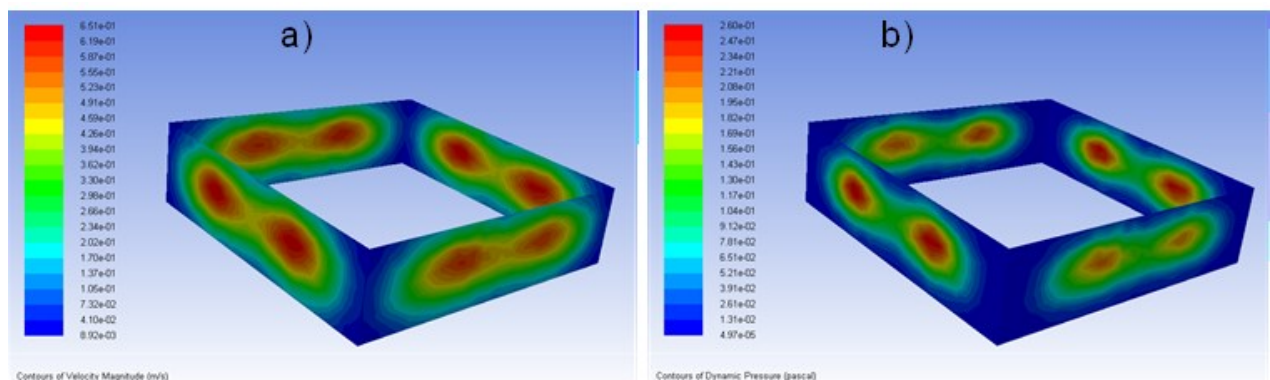

Fig. 10 Side surfaces: a) air velocity, b) dynamic pressure

Analyzing the distributions of the velocity and pressure fields, it can be concluded that the end of the turbulent flow zone is about $60 \mathrm{~mm}$ away from the limits of the computational domain (static zone). Taking this fact into account, the inlet of the air pollution measuring pipe should be located approx. $0.375 \mathrm{~m}$ from the geometrical center of the robot. However, due to the fact that states when the robot is tilted should also been taken into account, it would be a reasonable solution to place the inlet at a twice the distance $(0.75 \mathrm{~m})$, and 0.1 $\mathrm{m}$ below the axes of the propellers. 


\section{CONCLUSIONS}

The article presents experimental research carried out in laboratory conditions as well as modelling and results of simulation tests.

First, the quadrocopter motion equations have been developed, and then implemented in the Matlab/Simulink environment. In this way it was possible to simulate the various maneuvers of the robot: upward, forward, sideways and rotation flights. These models will be used further to select the structures and algorithms for controlling a quadrocopter flying robot.

Then, a dynamometer mobile station was designed and constructed, which made it possible to carry out measurements of the quadrocopter drive sets and determine their unknown parameters. The measurements were carried out for many different conditions: for various propellers, ESCs and various supply voltages (simulating battery discharge states).

The final stage of the work was to develop a numerical model and carry out CFD calculations. In this way, the pressure fields and velocities were determined for the quadrocopter flying robot. Another important aspect of this work was to verify the performance of the drive sets previously measured at the dynamometer station. The obtained results of simulation tests differ by $13 \%$ from the ones determined on the dynamometer. Based on the obtained velocity and pressure field distributions for the quadrocopter model, it was determined where the inlet of the air pollution measurement system should be located.

\section{REFERENCES}

Bolla, G. M., Casagrande, M., Comazzetto, A., et al. (2018). ARIA: Air Pollutants Monitoring Using UAVs. $5^{\text {th }}$ IEEE International Workshop on Metrology for AeroSpace (MetroAeroSpace), pp. 225-229.

Ciesielka, W., Suchanek, G. (2017). Project of flying robot type quadrocopter. Polish Journal of Environmental Studies, 26(5A), pp. 20-26.

Degner, M., Ewald, H. (2018). Mobile gas sensing system for detection of combustion pollutants - suitable fordrone based measurements. $12^{\text {th }}$ International Conference on Sensing Technology (ICST), pp. 39-44.

Shamsi, M. A., Shamsi, M. A., Dhaheri R. A., et al. (2018). Foggy Drone. Application to a Hexarotor UAV. Advances in Science and Engineering Technology International Conferences (ASET), pp.1-5.

Suchanek, G., Ciesielka, W. (2018). Design and experimental research of a quadrocopter flying robot, E3S Web of Conferences, 46(12), pp. 1-8,

Wang, L., Cavallaro A. (2018). Acoustic Sensing From a Multi-Rotor Drone, IEEE Sensors Journal, 18(11), pp. 4570-4582.

Wivou, J., Udawatta, L., Alshehhi A., et al. (2016). Quality Monitoring for Sustainable Systems via Drone Based Technology. IEEE International Conference on Information and Automation for Sustainability (ICIAfS), pp. 1-5. 


\begin{abstract}
.
In recent years, there has been a dynamic increase in the use of multirotor flying robots in various areas of economic and social life. Robots of this kind may be used in environmental research, after equipping them with an appropriate measuring systems. This includes taking measurements of various types of contaminants, such as: particulate matter (PM), various gases, noise and light pollution. To make this possible, it is necessary to conduct advanced model-simulation tests of the flying platform, analyse and determine the appropriate location for the measurement system. Most of the current research on methods and techniques of taking measurements on the flying platform does not take into account these issues. This work consists of two main parts: modeling and simulation tests, and experimental part carried out in laboratory conditions. As part of the work, quadrocopter dynamics equations have been developed and implemented in the Matlab/Simulink environment. The developed discrete mathematical model made it possible to simulate different robot maneuvers: upward, forward, sideways and rotation flight. In order to determine the required characteristics of the multicopter drive sets, a mobile dynamometer station was designed, constructed and programmed. The dynamometer allows, among others, to take measurements of thrust force, torque and rotational speed. The final stage of the work was the development of the numerical model and CFD calculations of the quadrocopter. In this part, distributions of the pressure fields and velocity for the robot's hover state were determined.
\end{abstract}

Keywords: quadrocopter, mathematical model, CFD simulation 